\title{
An efficient computational technique for local fractional heat conduction equations in fractal media
}

\author{
Duan Zhao a,b, Jagdev Singh ${ }^{c}$, Devendra Kumarc, Sushila Rathore ${ }^{d}$, Xiao-Jun Yange,f,* \\ aIOT Perception Mine Research Center, China University of Mining and Technology, Xuzhou 221008, China. \\ ${ }^{b}$ The National and Local Joint Engineering Laboratory of Internet Application Technology on Mine, Xuzhou 221008, China. \\ ${ }^{c}$ Department of Mathematics, JECRC University, Jaipur-303905, Rajasthan, India. \\ ${ }^{d}$ Department of Physics, Vivekananda Global University, Jaipur-303012, Rajasthan, India. \\ e School of Mechanics and Civil Engineering, China University of Mining and Technology, Xuzhou 221116, China. \\ ${ }^{f}$ State Key, Laboratory for Geomechanics and Deep Underground Engineering, China University of Mining and Technology, Xuzhou, \\ 221116, China.
}

Communicated by D. Baleanu

\begin{abstract}
The key aim of this article is to present an efficient numerical algorithm based on local fractional homotopy perturbation Sumudu transform technique for solving local fractional heat-conduction equations in fractal media. The proposed technique is an effective combination of local fractional homotopy perturbation method (LFHPM) and local fractional Sumudu transform algorithm. The results obtained by using the suggested scheme show that the approach is straightforward to apply and very accurate. (C) 2017 All rights reserved.
\end{abstract}

Keywords: Heat conduction equation, fractal media, local fractional derivative, local fractional homotopy perturbation method, local fractional Sumudu transform method.

2010 MSC: 35R11, 26A33, 35A15.

\section{Introduction}

In the field of science and engineering, fractals are used in many applications, such as fracture mechanics, nano fluids and porous media and so on. Since in nano-scale [10, 22] the various transport phenomena cannot be explained by smooth continuum approach, hence it is necessary to consider the fractal nature of the objects. In the thermal transport in a fractal bar, it is very important to describe the heat equations in many ways $[2,7,12-14,19,20,25,29,30,32,33]$.

Recent years, fractional calculus (FC) has gained significant attention and interest due to its wide applications. For example, Rayneau-Kirkhope et al. [13] discussed the ultra light fractals structures from hollow tubes. Shih [14] studied a literature survey on numerical heat transfer. Povestenko [12] analyzed

\footnotetext{
*Corresponding author

Email address: dyangxiaojun@163.com (Xiao-Jun Yang)
}

doi:10.22436/jnsa.010.04.17 
the fractional heat conduction equation and associated thermal stress. Hristov [7] explained the heatbalance integral to fractional heat diffusion sub-model. Wang et al. [20] represented the fractional model for the heat conduction in polar hairs.

More recently, local fractional calculus (LFC) [24, 26] was applied to discuss the non-differentiable problems, for instance, the local fractional heat-conduction [31-33], Tricomi [16], diffusion [9], damped and dissipative wave [18], Laplace [23] and Fokker-Planck equations [12], and electric circuit [27], etc.

The local fractional derivative operator is represented as follows $[3,8,16,18,23,24,26,27,31]$ :

$$
\frac{\partial^{\sigma} \theta(\xi, \eta)}{\partial \eta^{\sigma}}=\frac{\Delta^{\sigma}\left(\theta(\xi, \eta)-\theta\left(\xi, \eta_{0}\right)\right)}{\left(\eta-\eta_{0}\right)}
$$

where

$$
\Delta^{\sigma}\left(\theta(\xi, \eta)-\theta\left(\xi, \eta_{0}\right)\right) \cong \Gamma(1+\sigma)\left[\theta(\xi, \eta)-\theta\left(\xi, \eta_{0}\right)\right] .
$$

The aim of the present paper is to employ the local fractional homotopy perturbation Sumudu transform method (LFHPSTM) to solve the local fractional heat conduction equation occurring in fractal media with the local fractional derivative and initial conditions. The LFHPSTM is a combination of the local fractional homotopy perturbation method (LFHPM) [28] and Sumudu transform (ST) [17]. The LFHPM is a generalization of the classical homotopy perturbation method (HPM) [1,6].

The structure of this paper is as follows: In Section 2, the definitions of local fractional integrals and derivatives are discussed. In Section 3, the basic idea of the LFHPSTM is demonstrated. Section 4 presents the non-differentiable solutions for local fractional heat conduction equation. Finally, in Section 5 , the conclusions are discussed.

\section{Local fractional integrals and derivatives}

In this section, we present the basic theory of LFC, which is used in the present work.

Definition 2.1. Consider a function $\theta(\eta) \in C_{\sigma}(a, b)$, while

$$
\left|\theta(\eta)-\theta\left(\eta_{0}\right)\right|<\varepsilon^{\sigma}, \quad 0<\sigma \leqslant 1,
$$

is valid $[3,8,16,18,23,24,26,27,31]$, when $\left|\eta-\eta_{0}\right|<\delta$, for $\varepsilon, \delta>0$ and $\varepsilon \in \mathfrak{R}$.

Definition 2.2. Taking into consideration the interval $[a, b]$ and $\left(\xi_{j}, \xi_{j+1}\right), j=0, \cdots, N-1, \xi_{0}=a$ and $\xi_{N}=\mathrm{b}$ with $\Delta \xi_{j}=\xi_{j+1}-\xi_{j}, \Delta \xi=\max \left\{\Delta \xi_{0}, \Delta \xi_{1,}, \Delta \xi_{2,}, \cdots\right\}$ be partition of this interval. The local fractional integral of $\theta(\eta) \in C_{\sigma}(a, b)$ is represented as follows $[3,8,16,18,23,24,26,27,31]$ :

$$
\begin{aligned}
{ }_{a} \mathrm{I}_{\mathrm{b}}^{(\sigma)} \theta(\eta) & =\frac{1}{\Gamma(1+\sigma)} \int_{\mathrm{a}}^{\mathrm{b}} \theta(\xi)(\mathrm{d} \xi)^{\sigma} \\
& =\frac{1}{\Gamma(1+\sigma)} \operatorname{Lim}_{\Delta \xi \rightarrow 0} \sum_{j=0}^{j=N-1} \theta\left(\xi_{j}\right)\left(\Delta \xi_{j}\right)^{\sigma} .
\end{aligned}
$$

Definition 2.3. Let $\theta(\eta)$ satisfy the condition stated in (2.1), then the inverse formula of (2.2) is described as follows $[3,8,16,18,23,24,26,27,31]$ :

$$
\frac{d^{\sigma} \theta\left(\eta_{0}\right)}{d \eta^{\sigma}}=D_{\eta}^{(\sigma)} \theta\left(\eta_{0}\right)=\frac{\Delta^{\sigma}\left(\theta(\eta)-\theta\left(\eta_{0}\right)\right)}{\left(\eta-\eta_{0}\right)^{\sigma}}
$$

where

$$
\Delta^{\sigma}\left(\theta(\eta)-\theta\left(\eta_{0}\right)\right) \cong \Gamma(1+\sigma)\left[\theta(\eta)-\theta\left(\eta_{0}\right)\right]
$$


Definition 2.4. The local fractional ST of a function $\theta(\eta)$ is initiated and defined by Srivastava et al. [17] and represented in the form:

$$
\begin{aligned}
\operatorname{LFS}_{\sigma}\{\theta(\eta)\} & =\Theta_{\sigma}(z) \\
& =\frac{1}{\Gamma(1+\sigma)} \int_{0}^{\infty} E_{\sigma}\left(-z^{-\sigma} \eta^{\sigma}\right) \frac{\theta(\eta)}{z^{\sigma}}(d \eta)^{\sigma}, \quad 0<\sigma \leqslant 1 .
\end{aligned}
$$

The inverse formula is given as [17]:

$$
\operatorname{LFS}_{\sigma}^{-1}\left\{\Theta_{\sigma}(z)\right\}=\theta(\eta), \quad 0<\sigma \leqslant 1 .
$$

The ST was proposed by Watugala [21] and the further fundamental results were investigated by Belgacem et al. [4], Bulut et al. [5] and Singh et al. [15], etc.

Definition 2.5. The Mittag-Leffler function was introduced by Mittag-Leffler [11] and is represented as follows:

$$
E_{\sigma}(\eta)=\sum_{m=0}^{\infty} \frac{\eta^{k}}{\Gamma(\sigma m+1)}, \quad(\sigma \in C, \operatorname{Re}(\sigma)>0) .
$$

\section{Basic idea of the local fractional homotopy perturbation Sumudu transform method (LFHPSTM)}

In this section, we demonstrate an efficient computational technique based on the LFHPM [28, 32] and ST [17] scheme. In order to investigate the basic idea and solution process of the LFHPSTM, we have taken up the linear partial differential equation involving local fractional derivative:

$$
\mathrm{L}_{\sigma} \theta_{\sigma}(\xi, \eta)+R_{\sigma} \theta_{\sigma}(\xi, \eta)=h(\xi, \eta)
$$

where $L_{\sigma}$ indicates the linear local fractional differential operator, $R_{\sigma}$ denotes the remaining linear operator and $h(\xi, \eta)$ represents a non-homogenous function of $\xi$ and $\eta$ arising from source term.

On employing the local fractional ST on (3.1), it yields

$$
\begin{aligned}
\Theta_{\sigma}(z, \eta)= & \theta_{\sigma}(0, \eta)+z^{\sigma} \theta_{\sigma}^{\sigma}(0, \eta)+z^{2 \sigma} \theta_{\sigma}^{2 \sigma}(0, \eta)+\cdots \\
& +z^{(k-1) \sigma} \theta_{\sigma}^{(k-1) \sigma}(0, \eta)-z^{k \sigma} \operatorname{LFS}_{\sigma}\left[R_{\sigma} \theta_{\sigma}(\xi, \eta)\right]+z^{k \sigma} \operatorname{LFS}_{\sigma}[h(\xi, \eta)] .
\end{aligned}
$$

Applying the inverse of local fractional ST on (3.2), we get

$$
\begin{aligned}
\theta_{\sigma}(\xi, \eta)= & \theta_{\sigma}(0, \eta)+\frac{\eta^{\sigma}}{\Gamma(1+\sigma)} \theta_{\sigma}^{\sigma}(0, \eta)+\frac{\eta^{2 \sigma}}{\Gamma(1+2 \sigma)} \theta_{\sigma}^{2 \sigma}(0, \eta)+\cdots \\
& +\frac{\eta^{(k-1) \sigma}}{\Gamma(1+(k-1) \sigma)} \theta_{\sigma}^{(k-1) \sigma}(0, \eta)-\operatorname{LFS}_{\sigma}^{-1}\left[z^{k \sigma} \operatorname{LFS}_{\sigma}\left[R_{\sigma} \theta_{\sigma}(\xi, \eta)\right]\right] \\
& +\operatorname{LFS}_{\sigma}^{-1}\left[z^{k \sigma} \operatorname{LFS}_{\sigma}[h(\xi, \eta)]\right] .
\end{aligned}
$$

Now we apply the LFHPM $[28,32]$

$$
\theta_{\sigma}(\xi, \eta)=\sum_{m=0}^{\infty} p^{m \sigma} \theta_{\sigma, m}(\xi, \eta)
$$

By using (3.4) in (3.3), we get

$$
\begin{aligned}
\sum_{m=0}^{\infty} p^{m \sigma} \theta_{\sigma, m}(\xi, \eta)= & \theta_{\sigma}(0, \eta)+\frac{\eta^{\sigma}}{\Gamma(1+\sigma)} \theta_{\sigma}^{\sigma}(0, \eta)+\frac{\eta^{2 \sigma}}{\Gamma(1+2 \sigma)} \theta_{\sigma}^{2 \sigma}(0, \eta)+\cdots \\
& +\frac{\eta^{(k-1) \sigma}}{\Gamma(1+(k-1) \sigma)} \theta_{\sigma}^{(k-1) \sigma}(0, \eta)-p L F S_{\sigma}^{-1}\left[z^{k \sigma} \operatorname{LFS}_{\sigma}\left[R_{\sigma} \sum_{m=0}^{\infty} p^{m \sigma} \theta_{\sigma, m}(\xi, \eta)\right]\right] \\
& +\operatorname{LFS}_{\sigma}^{-1}\left[z^{k \sigma} \operatorname{LFS}_{\sigma}[h(\xi, \eta)]\right] .
\end{aligned}
$$


The above result is obtained by combining the local fractional ST [17] and LFHPM [28, 32].

Now by equating the coefficients of likewise powers of $p$, we have

$$
\begin{aligned}
p^{0 \sigma}: \theta_{\sigma, 0}(\xi, \eta)= & \theta_{\sigma}(0, \eta)+\frac{\eta^{\sigma}}{\Gamma(1+\sigma)} \theta_{\sigma}^{\sigma}(0, \eta)+\frac{\eta^{2 \sigma}}{\Gamma(1+2 \sigma)} \theta_{\sigma}^{2 \sigma}(0, \eta)+\cdots \\
& +\frac{\eta^{(k-1) \sigma}}{\Gamma(1+(k-1) \sigma)} \theta_{\sigma}^{(k-1) \sigma}(0, \eta)+\operatorname{LFS}_{\sigma}^{-1}\left[z^{k \sigma} \operatorname{LFS}_{\sigma}[h(\xi, \eta)]\right] \\
p^{1 \sigma}: \theta_{\sigma, 1}(\xi, \eta)= & -\operatorname{LFS}_{\sigma}^{-1}\left[z^{k \sigma} \operatorname{LFS}_{\sigma}\left[R_{\sigma} \theta_{\sigma, 0}(\xi, \eta)\right]\right] \\
p^{2 \sigma}: \theta_{\sigma, 2}(\xi, \eta)= & -\operatorname{LFS}_{\sigma}^{-1}\left[z^{k \sigma} \operatorname{LFS}_{\sigma}\left[R_{\sigma} \theta_{\sigma, 1}(\xi, \eta)\right]\right]
\end{aligned}
$$

Thus, series solution of local fraction differential (3.1) is expressed as follows:

$$
\theta_{\sigma}(\xi, \eta)=\lim _{M \rightarrow \infty} \sum_{m=0}^{M} \theta_{\sigma, m}(\xi, \eta) .
$$

\section{Non-differential solutions for local fractional heat-conduction equations}

In this section, we obtain the non-differential solutions for the linear heat conduction equations by using the combination of the LFHPM and local fractional ST operator.

Example 4.1. We consider the following linear local fractional heat conduction equation associated with local fractional derivative along with the initial conditions

$$
\begin{aligned}
\frac{\partial^{2 \sigma} \theta_{\sigma}(\xi, \eta)}{\partial \xi^{2 \sigma}}-\frac{\partial^{\sigma} \theta_{\sigma}(\xi, \eta)}{\partial \eta^{\sigma}} & =0, \quad \xi>0, \quad \eta>0 \\
\theta_{\sigma}(0, \eta) & =0 \\
\frac{\partial^{\sigma} \theta_{\sigma}(0, \eta)}{\partial \xi^{\sigma}} & =E_{\sigma}\left(\eta^{\sigma}\right) .
\end{aligned}
$$

By applying the local fractional ST on (4.1), we have

$$
\Theta_{\sigma}(z, \eta)=z^{\sigma} E_{\sigma}\left(\eta^{\sigma}\right)+z^{2 \sigma} \operatorname{LFS}_{\sigma}\left[\frac{\partial^{\sigma} \theta_{\sigma}(\xi, \eta)}{\partial \eta^{\sigma}}\right]
$$

Employing the inverse of local fractional ST on (4.2), we easily get the following formulation:

$$
\begin{aligned}
\theta_{\sigma}(\xi, \eta)= & \frac{\xi^{\sigma}}{\Gamma(1+\sigma)} E_{\sigma}\left(\eta^{\sigma}\right) \\
& +\operatorname{LFS}_{\sigma}^{-1}\left[z^{2 \sigma} \operatorname{LFS}_{\sigma}\left[\frac{\partial^{\sigma} \theta_{\sigma}(\xi, \eta)}{\partial \eta^{\sigma}}\right]\right] .
\end{aligned}
$$

Now by applying LFHPM [28, 32], we get

$$
\sum_{m=0}^{\infty} p^{m \sigma} \theta_{\sigma, m}(\xi, \eta)=\frac{\xi^{\sigma}}{\Gamma(1+\sigma)} E_{\sigma}\left(\eta^{\sigma}\right)+p L F S_{\sigma}^{-1}\left[z^{2 \sigma} \operatorname{LFS}_{\sigma}\left[\frac{\partial^{\sigma}\left(\sum_{m=0}^{\infty} p^{m \sigma} \theta_{\sigma, m}(\xi, \eta)\right)}{\partial \eta^{\sigma}}\right]\right] \text {. }
$$


Making the comparison of the coefficients of the power-like orders of $\mathrm{p}$, we get the following components of the series solution

$$
\begin{aligned}
& p^{0 \sigma}: \theta_{\sigma, 0}(\xi, \eta)=\frac{\xi^{\sigma}}{\Gamma(1+\sigma)} E_{\sigma}\left(\eta^{\sigma}\right), \\
& p^{1 \sigma}: \theta_{\sigma, 1}(\xi, \eta)=\frac{\xi^{3 \sigma}}{\Gamma(1+3 \sigma)} E_{\sigma}\left(\eta^{\sigma}\right), \\
& p^{2 \sigma}: \theta_{\sigma, 2}(\xi, \eta)=\frac{\xi^{5 \sigma}}{\Gamma(1+5 \sigma)} E_{\sigma}\left(\eta^{\sigma}\right),
\end{aligned}
$$

and so on. By appealing the same way, we obtain the remaining iterates of the approximate solution. Thus, the solution of local fractional heat-conduction equation (4.1) is expressed as:

$$
\begin{aligned}
\theta_{\sigma}(\xi, \eta) & =\sum_{m=0}^{\infty} \theta_{\sigma, m}(\xi, \eta) \\
& =E_{\sigma}\left(\eta^{\sigma}\right)\left[\frac{\xi^{\sigma}}{\Gamma(1+\sigma)}+\frac{\xi^{3 \sigma}}{\Gamma(1+3 \sigma)}+\frac{\xi^{5 \sigma}}{\Gamma(1+5 \sigma)}+\cdots\right] .
\end{aligned}
$$

It can also be presented in closed form as

$$
\theta_{\sigma}(\xi, \eta)=E_{\sigma}\left(\eta^{\sigma}\right) \sum_{m=0}^{\infty} \frac{\xi^{(2 m+1) \sigma}}{\Gamma[1+(2 m+1) \sigma]} .
$$

Example 4.2. Let us consider the nonhomogeneous local fractional heat equation associated with local fractional derivative along with the initial conditions given as follows:

$$
\begin{gathered}
\frac{\partial^{\sigma} \theta_{\sigma}(\xi, \eta)}{\partial \eta^{\sigma}}-\frac{\partial^{2 \sigma} \theta_{\sigma}(\xi, \eta)}{\partial \xi^{2 \sigma}}=-\frac{\xi^{\sigma}}{\Gamma(1+\sigma)} E_{\sigma}\left(-\eta^{\sigma}\right), \quad \xi>0, \quad \eta>0, \\
\theta_{\sigma}(0, \eta)=0, \\
\frac{\partial^{\sigma} \theta_{\sigma}(0, \eta)}{\partial \xi^{\sigma}}=E_{\sigma}\left(-\eta^{\sigma}\right) .
\end{gathered}
$$

Using the local fractional ST on (4.3), we obtain

$$
\Theta_{\sigma}(z, \eta)=z^{\sigma} E_{\sigma}\left(-\eta^{\sigma}\right)+z^{2 \sigma} \operatorname{LFS}_{\sigma}\left[\frac{\partial^{\sigma} \theta_{\sigma}(\xi, \eta)}{\partial \eta^{\sigma}}\right]+z^{2 \sigma} \operatorname{LFS}_{\sigma}\left[\frac{\xi^{\sigma}}{\Gamma(1+\sigma)} E_{\sigma}\left(-\eta^{\sigma}\right)\right] .
$$

By applying the inverse of local fractional ST on (4.4), we have

$$
\begin{aligned}
\theta_{\sigma}(\xi, \eta)= & \frac{\xi^{\sigma}}{\Gamma(1+\sigma)} E_{\sigma}\left(-\eta^{\sigma}\right)+\operatorname{LFS}_{\sigma}^{-1}\left[z^{2 \sigma} \operatorname{LFS}_{\sigma}\left[\frac{\partial^{\sigma} \theta_{\sigma}(\xi, \eta)}{\partial \eta^{\sigma}}\right]\right] \\
& +\operatorname{LFS}_{\sigma}^{-1}\left[z^{2 \sigma} \operatorname{LFS}_{\sigma}\left[\frac{\xi^{\sigma}}{\Gamma(1+\sigma)} E_{\sigma}\left(-\eta^{\sigma}\right)\right]\right] .
\end{aligned}
$$

Now employing LFHPM [28, 32], we get

$$
\begin{aligned}
\sum_{m=0}^{\infty} p^{m \sigma} \theta_{\sigma, m}(\xi, \eta)= & \frac{\xi^{\sigma}}{\Gamma(1+\sigma)} E_{\sigma}\left(-\eta^{\sigma}\right)+p \operatorname{LFS}_{\sigma}^{-1}\left[z^{2 \sigma} \operatorname{LFS}_{\sigma}\left[\frac{\partial^{\sigma}\left(\sum_{m=0}^{\infty} p^{m \sigma} \theta_{\sigma, m}(\xi, \eta)\right)}{\partial \eta^{\sigma}}\right]\right] \\
& +\operatorname{LFS}_{\sigma}^{-1}\left[z^{2 \sigma} \operatorname{LFS}_{\sigma}\left[\frac{\xi^{\sigma}}{\Gamma(1+\sigma)} E_{\sigma}\left(-\eta^{\sigma}\right)\right]\right] .
\end{aligned}
$$


Taking into account the coefficients of the power-like orders of $\mathrm{p}$, we obtain the following components of the series solution

$$
\begin{aligned}
& p^{0 \sigma}: \theta_{\sigma, 0}(\xi, \eta)=\frac{\xi^{\sigma}}{\Gamma(1+\sigma)} E_{\sigma}\left(-\eta^{\sigma}\right)+\frac{\xi^{3 \sigma}}{\Gamma(1+3 \sigma)} E_{\sigma}\left(-\eta^{\sigma}\right), \\
& p^{1 \sigma}: \theta_{\sigma, 1}(\xi, \eta)=-\frac{\xi^{3 \sigma}}{\Gamma(1+3 \sigma)} E_{\sigma}\left(-\eta^{\sigma}\right)-\frac{\xi^{5 \sigma}}{\Gamma(1+5 \sigma)} E_{\sigma}\left(-\eta^{\sigma}\right), \\
& p^{2 \sigma}: \theta_{\sigma, 2}(\xi, \eta)=+\frac{\xi^{5 \sigma}}{\Gamma(1+5 \sigma)} E_{\sigma}\left(-\eta^{\sigma}\right)+\frac{\xi^{7 \sigma}}{\Gamma(1+7 \sigma)} E_{\sigma}\left(-\eta^{\sigma}\right),
\end{aligned}
$$

Thus, the solution of local fractional heat conduction equation (4.3) is presented as follows:

$$
\begin{aligned}
\theta_{\sigma}(\xi, \eta) & =\sum_{m=0}^{\infty} \theta_{\sigma, m}(\xi, \eta) \\
& =E_{\sigma}\left(-\eta^{\sigma}\right)\left[\frac{\xi^{\sigma}}{\Gamma(1+\sigma)}+\frac{\xi^{3 \sigma}}{\Gamma(1+3 \sigma)}-\frac{\xi^{3 \sigma}}{\Gamma(1+3 \sigma)}-\frac{\xi^{5 \sigma}}{\Gamma(1+5 \sigma)}+\frac{\xi^{5 \sigma}}{\Gamma(1+5 \sigma)} \cdots\right] .
\end{aligned}
$$

Finally, we obtain the solution in closed form as

$$
\theta_{\sigma}(\xi, \eta)=E_{\sigma}\left(-\eta^{\sigma}\right) \frac{\xi^{\sigma}}{\Gamma(1+\sigma)} .
$$

Example 4.3. Consider the nonhomogeneous local fractional heat equation associated with local fractional derivative

$$
\frac{\partial^{\sigma} \theta_{\sigma}(\xi, \eta)}{\partial \eta^{\sigma}}-\frac{\partial^{2 \sigma} \theta_{\sigma}(\xi, \eta)}{\partial \xi^{2 \sigma}}=\frac{\xi^{\sigma}}{\Gamma(1+\sigma)} \cos _{\sigma}\left(\eta^{\sigma}\right), \quad \xi>0, \quad \eta>0
$$

with the initial conditions given as follows:

$$
\begin{aligned}
\theta_{\sigma}(0, \eta) & =0, \\
\frac{\partial^{\sigma} \theta_{\sigma}(0, \eta)}{\partial \xi^{\sigma}} & =\sin _{\sigma}\left(\eta^{\sigma}\right) .
\end{aligned}
$$

By employing local fractional ST on (4.5), we have the following:

$$
\begin{aligned}
\Theta_{\sigma}(z, \eta)= & z^{\sigma} \sin _{\sigma}\left(\eta^{\sigma}\right)+z^{2 \sigma} \operatorname{LFS}_{\sigma}\left[\frac{\partial^{\sigma} \theta_{\sigma}(\xi, \eta)}{\partial \eta^{\sigma}}\right] \\
& -z^{2 \sigma} \operatorname{LFS}_{\sigma}\left[\frac{\xi^{\sigma}}{\Gamma(1+\sigma)} \cos _{\sigma}\left(\eta^{\sigma}\right)\right] .
\end{aligned}
$$

Using the inverse of local fractional ST on (4.6), we get

Now applying LFHPM [28, 32], we obtain that

$$
\begin{aligned}
\theta_{\sigma}(\xi, \eta)= & \frac{\xi^{\sigma}}{\Gamma(1+\sigma)} \sin _{\sigma}\left(\eta^{\sigma}\right)+\operatorname{LFS}_{\sigma}^{-1}\left[z^{2 \sigma} \operatorname{LFS}_{\sigma}\left[\frac{\partial^{\sigma} \theta_{\sigma}(\xi, \eta)}{\partial \eta^{\sigma}}\right]\right] \\
& -\operatorname{LFS}_{\sigma}^{-1}\left[z^{2 \sigma} \operatorname{LFS}_{\sigma}\left[\frac{\xi^{\sigma}}{\Gamma(1+\sigma)} \cos _{\sigma}\left(\eta^{\sigma}\right)\right]\right]
\end{aligned}
$$

$$
\begin{aligned}
\sum_{m=0}^{\infty} p^{m \sigma} \theta_{\sigma, m}(\xi, \eta)= & \frac{\xi^{\sigma}}{\Gamma(1+\sigma)} \sin _{\sigma}\left(\eta^{\sigma}\right)+\operatorname{pLF}_{\sigma}^{-1}\left[z^{2 \sigma} \operatorname{LFS}_{\sigma}\left[\frac{\partial^{\sigma}\left(\sum_{m=0}^{\infty} p^{m \sigma} \theta_{\sigma, m}(\xi, \eta)\right)}{\partial \eta^{\sigma}}\right]\right] \\
& -\operatorname{LFS}_{\sigma}^{-1}\left[z^{2 \sigma} \operatorname{LFS}_{\sigma}\left[\frac{\xi^{\sigma}}{\Gamma(1+\sigma)} \cos _{\sigma}\left(\eta^{\sigma}\right)\right]\right]
\end{aligned}
$$


Comparing the coefficients of the power-like orders of $\mathrm{p}$, we get the following components of the series solution

$$
\begin{aligned}
& p^{0 \sigma}: \theta_{\sigma, 0}(\xi, \eta)=\frac{\xi^{\sigma}}{\Gamma(1+\sigma)} \sin _{\sigma}\left(\eta^{\sigma}\right)-\frac{\xi 3^{\sigma}}{\Gamma(1+3 \sigma)} \cos _{\sigma}\left(\eta^{\sigma}\right), \\
& p^{1 \sigma}: \theta_{\sigma, 1}(\xi, \eta)=\frac{\xi^{3 \sigma}}{\Gamma(1+3 \sigma)} \cos _{\sigma}\left(\eta^{\sigma}\right)+\frac{\xi^{5 \sigma}}{\Gamma(1+5 \sigma)} \sin _{\sigma}\left(\eta^{\sigma}\right), \\
& p^{2 \sigma}: \theta_{\sigma, 2}(\xi, \eta)=-\frac{\xi^{5 \sigma}}{\Gamma(1+5 \sigma)} \sin _{\sigma}\left(\eta^{\sigma}\right)+\frac{\xi^{7 \sigma}}{\Gamma(1+7 \sigma)} \cos _{\sigma}\left(\eta^{\sigma}\right),
\end{aligned}
$$

Hence, the solution of local fractional heat conduction equation (4.5) is given as follows:

$$
\begin{aligned}
\theta_{\sigma}(\xi, \eta)= & \sum_{m=0}^{\infty} \theta_{\sigma, m}(\xi, \eta) \\
= & \frac{\xi^{\sigma}}{\Gamma(1+\sigma)} \sin _{\sigma}\left(\eta^{\sigma}\right)+\cos _{\sigma}\left(\eta^{\sigma}\right) \frac{\xi^{3 \sigma}}{\Gamma(1+3 \sigma)}-\cos _{\sigma}\left(\eta^{\sigma}\right) \frac{\xi^{3 \sigma}}{\Gamma(1+3 \sigma)} \\
& +\sin _{\sigma}\left(\eta_{\sigma}\right) \frac{\xi^{5 \sigma}}{\Gamma(1+5 \sigma)}-\frac{\xi^{5 \sigma}}{\Gamma(1+5 \sigma)} \sin _{\sigma}\left(\eta^{\sigma}\right)+\frac{\xi^{7 \sigma}}{\Gamma(1+7 \sigma)} \cos _{\sigma}\left(\eta^{\sigma}\right) \cdots
\end{aligned}
$$

Consequently, we get the solution in closed form as

$$
\theta_{\sigma}(\xi, \eta)=\frac{\xi^{\sigma}}{\Gamma(1+\sigma)} \sin _{\sigma}\left(\eta^{\sigma}\right)
$$

Example 4.4. Finally, let us consider the nonhomogeneous local fractional heat conduction equation associated with local fractional derivative along with the initial conditions given as follows:

$$
\begin{gathered}
\frac{\partial^{\sigma} \theta_{\sigma}(\xi, \eta)}{\partial \eta^{\sigma}}-\frac{\partial^{2 \sigma} \theta_{\sigma}(\xi, \eta)}{\partial \xi^{2 \sigma}}=1, \quad \xi>0, \quad \eta>0, \\
\theta_{\sigma}(0, \eta)=\frac{\eta^{\sigma}}{\Gamma(1+\sigma)^{\prime}}, \\
\frac{\partial^{\sigma} \theta_{\sigma}(0, \eta)}{\partial \xi^{\sigma}}=0 .
\end{gathered}
$$

By putting up the local fractional ST on (4.7), we get

$$
\Theta_{\sigma}(z, \eta)=\frac{\eta^{\sigma}}{\Gamma(1+\sigma)}-z^{2 \sigma}+z^{2 \sigma} \operatorname{LFS}_{\sigma}\left[\frac{\partial^{\sigma} \theta_{\sigma}(\xi, \eta)}{\partial \eta^{\sigma}}\right]
$$

Using the inverse of local fractional ST on (4.8), we have the following:

$$
\begin{aligned}
\theta_{\sigma}(\xi, \eta)= & \frac{\xi^{\sigma}}{\Gamma(1+\sigma)}-\frac{\xi^{2 \sigma}}{\Gamma(1+2 \sigma)} \\
& +\operatorname{LFS}_{\sigma}^{-1}\left[z^{2 \sigma} \operatorname{LFS}_{\sigma}\left[\frac{\partial^{\sigma} \theta_{\sigma}(\xi, \eta)}{\partial \eta^{\sigma}}\right]\right] .
\end{aligned}
$$

By applying LFHPM [28, 32], we get

$$
\sum_{m=0}^{\infty} p^{m \sigma} \theta_{\sigma, m}(\xi, \eta)=\frac{\xi^{\sigma}}{\Gamma(1+\sigma)}+\operatorname{pLFS}_{\sigma}^{-1}\left[z^{2 \sigma} \operatorname{LFS}_{\sigma}\left[\frac{\partial^{\sigma}\left(\sum_{m=0}^{\infty} p^{m \sigma} \theta_{\sigma, m}(\xi, \eta)\right)}{\partial \eta^{\sigma}}\right]\right]
$$


Considering the comparison of the coefficients of the power-like orders of $p$, we obtain the following components of the series solution

$$
\begin{aligned}
& p^{0 \sigma}: \theta_{\sigma, 0}(\xi, \eta)=\frac{\eta^{\sigma}}{\Gamma(1+\sigma)}-\frac{\xi^{2 \sigma}}{\Gamma(1+2 \sigma)} \\
& p^{1 \sigma}: \theta_{\sigma, 1}(\xi, \eta)=\frac{\xi^{2 \sigma}}{\Gamma(1+2 \sigma)} \\
& p^{2 \sigma}: \theta_{\sigma, 2}(\xi, \eta)=0
\end{aligned}
$$

Thus, the solution of local fractional heat conduction equation (4.7) is expressed as:

$$
\begin{aligned}
\theta_{\sigma}(\xi, \eta) & =\sum_{m=0}^{\infty} \theta_{\sigma, m}(\xi, \eta) \\
& =\frac{\eta^{\sigma}}{\Gamma(1+\sigma)}-\frac{\xi^{2 \sigma}}{\Gamma(1+2 \sigma)}+\frac{\xi^{2 \sigma}}{\Gamma(1+2 \sigma)}+0+\cdots
\end{aligned}
$$

Finally, we get the exact solution in the form:

$$
\theta_{\sigma}(\xi, \eta)=\frac{\eta^{\sigma}}{\Gamma(1+\sigma)}
$$

\section{Conclusions}

In this work the local fractional heat-conduction equations arising in fractal media have been investigated. The LFHPSTM is applied to find the non-differentiable solutions of the heat-conduction equations in fractal media. For the various real world problems, the introduced computational technique is very simple, easy to employ and computationally very attractive. Thus, we can conclude that the proposed technique is a powerful tool for solving the local fractional linear equations arising in fractal media.

\section{Acknowledgment}

The work was supported by supported by the Joint Prospective Innovation Fund research of Jiangsu Province (No.BY2015023-04) and the National Basic Research Program of China (973 Program) (No.2014CB046305).

\section{References}

[1] A. Atangana, Extension of the Sumudu homotopy perturbation method to an attractor for one-dimensional Keller-Segel equations, Appl. Math. Model., 39 (2015), 2909-2916. 1

[2] A. Atangana, D. Baleanu, New fractional derivatives with non-local and non-singular kernel: Theory and application to heat transfer model, Therm. Sci., 20 (2016), 763-769. 1

[3] D. Baleanu, H. M. Srivastava, X.-J. Yang, Local fractional variational iteration algorithms for the parabolic Fokker-Planck equation defined on Cantor sets, Prog. Fract. Differ. Appl., 1 (2015), 1-11. 1, 2.1, 2.2, 2.3

[4] F. B. M. Belgacem, A. A. Karaballi, S. L. Kalla, Analytical investigations of the Sumudu transform and applications to integral production equations, Math. Probl. Eng., 3 (2003), 103-118. 2

[5] H. Bulut, H. M. Baskonus, S. Tuluce, The solutions of partial differential equations with variable coefficient by Sumudu transform method, AIP Conf. Proc., 1493 (2012), 91-95. 2

[6] J.-H. He, Homotopy perturbation technique, Comput. Methods Appl. Mech. Engrg., 178 (1999), 257-262. 1

[7] J. Hristov, Heat-balance integral to fractional (half-time) heat diffusion sub-model, Therm. Sci., 14 (2010), 291-316. 1

[8] H. Jafari, H. K. Jassim, S. P. Moshokoa, V. M. Ariyan, F. Tchier, Reduced differential transform method for partial differential equations within local fractional derivative operators, Adv. Mech. Eng., 8 (2016), 1-6. 1, 2.1, $2.2,2.3$ 
[9] M. Ma, D. Baleanu, Y. S. Gasimov, X.-J. Yang, New results for multidimensional diffusion equations in fractal dimensional space, Rom. J. Phys., 61 (2016), 784-794. 1

[10] M. Majumder, N. Chopra, R. Andrews, B. J. Hinds, Nanoscale hydrodynamics: enhanced flow in carbon nanotubes, Nature, 438 (2005), 44-44. 1

[11] G. M. Mittag-Leffler, Sur la nouvelle fonction $E_{\alpha}(x)$, CR Acad. Sci. Paris, 137 (1903), 554-558. 2.5

[12] Y. Z. Povstenko, Fractional heat conduction equation and associated thermal stress, J. Therm. Stresses, 28 (2004), 83-102. 1

[13] D. Rayneau-Kirkhope, Y. Mao, R. Farr, Ultralight fractal structures from hollow tubes, Phys. Rev. Lett., 109 (2012), 204301-204304. 1

[14] T.-M. Shih, A literature survey on numerical heat transfer, Num. Heat Transfer, 5 (1982), 369-420. 1

[15] J. Singh, D. Kumar, A. Kılıçman, Numerical solutions of nonlinear fractional partial differential equations arising in spatial diffusion of biological populations, Abstr. Appl. Anal., 2014 (2014), 12 pages. 2

[16] J. Singh, D. Kumar, J. J. Nieto, A reliable algorithm for a local fractional Tricomi equation arising in fractal transonic flow, Entropy, 18 (2016), 8 pages. 1, 2.1, 2.2, 2.3

[17] H. M. Srivastava, A. K. Golmankhaneh, D. Baleanu, X .J. Yang, Local fractional Sumudu transform with application to IVPs on Cantor sets, Abstr. Appl. Anal., 2014 (2014), 7 pages. 1, 2.4, 3, 3

[18] W.-H. Su, D. Baleanu, X.-J. Yang, H. Jafari, Damped wave equation and dissipative wave equation in fractal strings within the local fractional variational iteration method, Fixed Point Theory Appl., 2013 (2013), 11 pages. 1, 2.1, $2.2,2.3$

[19] V. E. Tarasov, Heat transfer in fractal materials, Int. J. Heat Mass Transfer, 93 (2016), 427-430. 1

[20] Q.-L. Wang, J.-H. He, Z.-B. Li, Fractional model for heat conduction in polar bear hairs, Therm. Sci., 16 (2012), $339-342$. 1

[21] G. K. Watugala, Sumudu transform: a new integral transform to solve differential equations and control engineering problems, Internat. J. Math. Ed. Sci. Tech., 24 (1993), 35-43. 2

[22] Y.-M. Xuan, W. Roetzel, Conceptions for heat transfer correlation of nanofluids, Int. J. Heat Mass Transfer, 43 (2000), 3701-3707. 1

[23] S.-P. Yan, H. Jafari, H. K. Jassim, Local fractional Adomian decomposition and function decomposition methods for Laplace equation within local fractional operators, Adv. Math. Phys., 2014 (2014), 7 pages. 1, 2.1, 2.2, 2.3

[24] X.-J. Yang, Advanced local fractional calculus and its applications, World Science, New York, NY, USA, (2012). 1, 2.1, $2.2,2.3$

[25] X.-J. Yang, D. Baleanu, Fractal heat conduction problem solved by local fractional variation iteration method, Therm. Sci., 17 (2013), 625-628. 1

[26] X.-J. Yang, D. Baleanu, H. M. Srivastava, Local fractional integral transforms and their applications, Academic Press, (2015). 1, 2.1, 2.2, 2.3

[27] X.-J. Yang, J. A. T. Machado, C. Cattani, F. Gao, On a fractal LC-electric circuit modeled by local fractional calculus, Commun. Nonlinear Sci. Numer. Simul., 47 (2017), 200-206. 1, 2.1, 2.2, 2.3

[28] X.-J. Yang, H. M. Srivastava, C. Cattani, Local fractional homotopy perturbation method for solving fractal partial differential equations arising in mathematical physics, Rom. Rep. Phys., 67 (2015), 752-761. 1, 3, 3, 3, 4.1, 4.2, 4.3, 4.4

[29] X.-J. Yang, H. M. Srivastava, J.-H. He, D. Baleanu, Cantor-type cylindrical-coordinate method for differential equations with local fractional derivatives, Phys. Lett. A, 377 (2013), 1696-1700. 1

[30] X.-J. Yang, H. M. Srivastava, J. A. T. Machado, A new fractional derivative without singular kernel: Application to the modelling of the steady heat flow, Therm. Sci., 20 (2016), 753-756. 1

[31] A.-M. Yang, Y.-Z. Zhang, Y. Long, The Yang-Fourier transforms to heat-conduction in a semi-infinite fractal bar, Therm. Sci., 17 (2013), 707-713. 1, 2.1, 2.2, 2.3

[32] Y. Zhang, C. Cattani, X.-J. Yang, Local fractional homotopy perturbation method for solving non-homogeneous heat conduction equations in fractal domains, Entropy, 17 (2015), 6753-6764. 1, 3, 3, 3, 4.1, 4.2, 4.3, 4.4

[33] D. Zhao, X.-J. Yang, H. M. Srivastava, On the fractal heat transfer problems with local fractional calculus, Therm. Sci., 19 (2015), 1867-1871. 1 